\title{
XV. Nanocatalysis
}

Catalysis is a phenomenon in which a small quantity of a substance, called the catalyst, increases substantially the rate of a chemical or a biological reaction without being consumed. Many chemical and biological processes rely on catalysis. The Swedish chemist J.J.Berzelius was the first, in 1835 , to notice that certain chemicals accelerate chemical reactions while remaining unchanged after the reaction. Today, $90 \%$ of all commercial chemical processes rely on catalysis.

\section{Catalysts make life easier}

The principle of catalysis is schematically shown in figure 120 . The initial and final states of a reaction are separated by a potential barrier. The higher and thicker the barrier, the slower is the speed of reaction. The role of a catalyst is to reduce this barrier and make the reaction easier to process.

A catalyst changes the way the reaction is performed, but not a reactant or final product. It modifies the speed of a reaction, its pathway and can guide the reactants towards the production of a specific product.

The lower the activation energy (the height of the barrier in figure 120) the lower is the temperature required for a reaction to occur at a reasonable rate. For example, suppose that a reaction takes place at a temperature of about $1000 \mathrm{~K}$ without a catalyst and that the initial activation energy is $250 \mathrm{~kJ} / \mathrm{mole}$. If a catalyst decreases the activation to $100 \mathrm{~kJ} / \mathrm{mole}$, then the reaction can be carried out around $400 \mathrm{~K}$.

In organic reactions, there are four main types of catalysis used to accelerate chemical reactions: acid catalysis, base catalysis, nucleophilic catalysis and metal-ion catalysis. To illustrate the efficiency of a catalyst in a chemical reaction, we can consider the reaction $\mathrm{C}_{2} \mathrm{H}_{4}+\mathrm{H}_{2} \rightarrow \mathrm{C}_{2} \mathrm{H}_{6}$ in which ethylene is hydrogenated into ethane. Performing this reaction at $300 \mathrm{~K}$ with Platinum as a catalyst enhances the rate of the reaction by a factor $10^{42}$ !

Catalysis can be either homogeneous or heterogeneous (figure 121). In homogeneous catalysis, the reactants and the catalyst are in the same phase. This is not the case in heterogeneous catalysis. The main advantage of heterogeneous catalysis is that the catalyst can be easily used again. Compared to homogeneous catalysis, heterogeneous catalysis is generally cheaper and more selective. However, on a per catalyst atom basis, homogeneous 


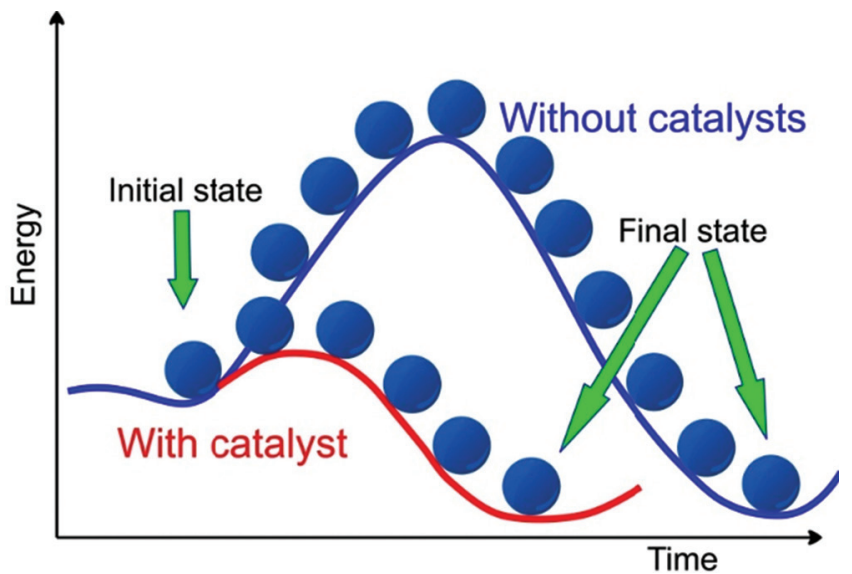

Figure 120. Principle of catalysis.

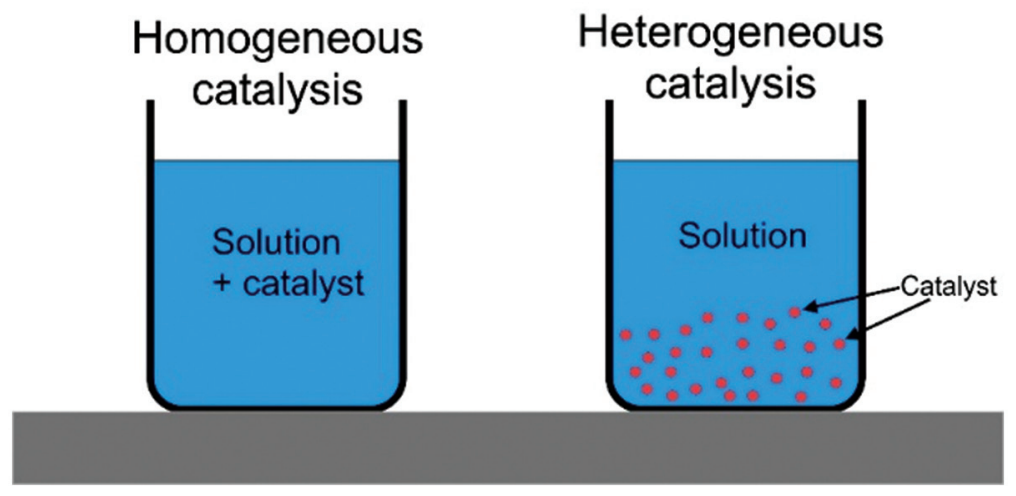

Figure 121. Difference between homogeneous and heterogeneous catalysis.

catalysis is usually more efficient than heterogeneous catalysis. Examples of families of homogeneous catalysts are: acids, bases, metal salts, radical initiators, enzymes. Examples of families of heterogeneous catalysts are: supported metals, solid acids and bases, transition metal oxides and sulfides, immobilized enzymes, polymer bound species.

A good catalyst needs to have several properties which are shown in figure 122 .

It should be highly stable to resist the physical conditions of the reaction and have the possibility to be re-used several times. When several reactions are possible with the reactants, the catalyst should have a high selectivity to favor the desired reaction with respect to the others. While a catalyst cannot change the equilibrium yield of a reaction it can increase the practically achievable yield. An additional desirable feature for a catalyst is that 


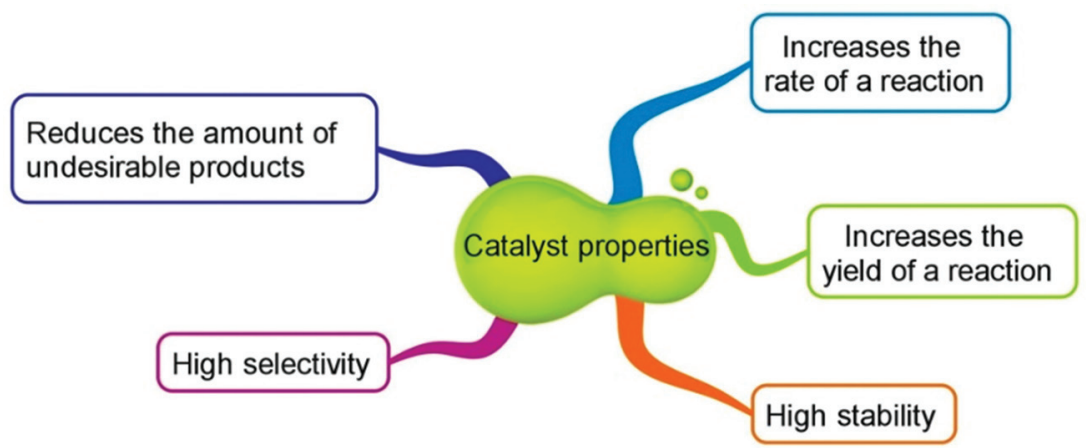

Figure 122. Properties that a good catalyst must have.

it reduces the number of undesirable products formed in the reaction, or helps to produce useful byproducts.

In heterogeneous catalysis, the catalyst is often deposited on a support, especially in the case of metals. The reason is that some metals, such as platinum, are expensive and only the surface is active. Therefore, platinum is deposited on a cheap support which gives also the strength to the whole catalyst system.

The efficiency of a given catalyst depends on several parameters: size, shape, support on which it is deposited... Since the interface between the reactants and the catalyst is one of the most relevant issues, it means that a good catalyst should have a large area of contact. This is precisely what nanoparticles offer, with a high surface area-to-volume ratio, and explains why the nanocatalysis domain is important.

\section{Nanocatalysts for chemical reactions}

Several nanomaterials are now used as catalysts. Some of them are indicated in figure 123 .

In homogeneous catalysis, transition metal nanoparticles are frequently used in the form of colloidal solutions. The solvent used can be water, an organic solvent or a mixture. These solutions must be stabilized in order to prevent aggregation of the nanoparticles otherwise the catalytic activity would drop. The choice of the stabilizer is therefore important to maintain a high reactivity for the catalyst.

The colloidal solution should be made in such a way that the catalyst can be easily recycled. The most frequently used method to synthesize colloidal 


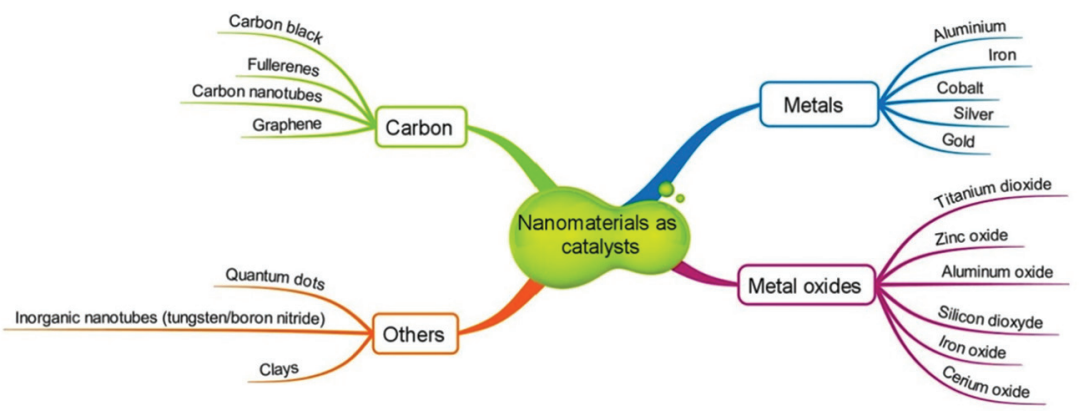

Figure 123. Some example of nanomaterials used as nanocatalysts.

solutions of transition metal nanoparticles is by chemical reduction of a metal salt. There are other synthetic methods that are used less frequently, i. e., thermal, photochemical or sonochemical reduction, ligand reduction, metal vapor synthesis or electrochemical reduction (details can be found at www.nanowerk.com).

In heterogeneous catalysis, the metal nanoparticles are linked onto a support. This requires either a functionalization of the support to adsorb nanoparticles or that the support be manufactured with lithographic techniques.

In addition, nanoparticles can be chemically more active than larger particles. For example, gold is a rather chemically inert metal. It is not corroded under normal external conditions. Surprisingly, it turns out that gold nanoparticles are chemically active and can be used as catalysts. The catalytic properties depend on the gold particle size $(<5 \mathrm{~nm})$, on the nature of the support, and on the contact structure. This last item is important because it governs the availability and order of the gold atoms. Gold nanoparticles are so active that they are able to catalyze carbon monoxide (CO) oxidation at temperatures far below $0^{\circ} \mathrm{C}$. They are also able to decompose gases such as sulfur dioxide which is an air-pollutant.

The Claisen-Schmidt condensation reaction is an aldol condensation of ketones and arylaldehydes to form unsaturated molecules. It is a route to produce molecules such as flavanone and its derivatives which are important intermediates in the synthesis of anticancer, anti-AIDS, anti-inflammatory and antibacterial drugs. This reaction can be catalyzed by $\mathrm{MgO}$. Nanocrystalline magnesium oxide (MgO) is often used as a catalyst ${ }^{11}$. Several crystalline forms are available with different catalytic activity. The less efficient form is commercial Mgo, denoted CM-MgO. It has a specific area ranging 
between 10 and $30 \mathrm{~m}^{2} / \mathrm{g}$. Other crystalline forms can be prepared such as the so-called conventionally prepared $\mathrm{MgO}(\mathrm{NA}-\mathrm{MgO})$ or the aerogel prepared $\mathrm{MgO}$ (NAP-MgO). They have a specific areas of $130-250 \mathrm{~m}^{2} / \mathrm{g}$ and $300-590 \mathrm{~m}^{2} / \mathrm{g}$, respectively. The percentage of ions on the edges, which is related to the reactivity of the catalyst is equal to $0.4 \%$ (CM-MgO), $0.5 \%$ (NA-MgO) and $21 \%$ (NAP-MgO).

It turns out that the yield and speed of the reaction depends very much of the crystalline form of MgO. With CM-MgO, a yield of $85 \%$ is obtained in $5^{0}$ hours. NA-Mgo is more efficient with $97 \%$ in 18 hours. However, the best results re obtained with NAP-MgO: $97 \%$ in 12 hours. Clearly, nanocatalysts are very efficient and their nanostructure plays an important role.

\section{Enzymes speed up biological reactions}

Most biological catalysts are enzymes. The names of enzymes usually end with the suffix "ase" indicating a bond breaking. For example, a peptidase breaks a peptide bond in a protein. An esterase breaks an ester bond, transforming the ester into an acid and an alcohol. The word synthase, on the contrary, means synthesizing instead of breaking. The ATP (adenosine triphosphate, considered as the "energy currency of life") synthase, for example, catalyzes the synthesis of ATP.

There are several models explaining enzyme-substrate binding. Two of them are displayed schematically in figure 124 :

- The lock-and-key model, proposed at the end of the nineteen century by E.Fischer, supposes that there is a perfect fit between the shape of the substrate and that of the enzyme. This is similar to what happens with a key and a lock.

- In the induced-fit model the configuration of the active site changes to fit that of the substrate.

Compared to man-made catalysts used to speed up chemical reactions, enzymes are highly complex molecules. This is illustrated by trypsin, a medium size globular protein that breaks peptide bonds present in other proteins. The mechanism by which the bond breaking occurs is described in the following web site: http://www.bb.iastate.edu/ thorn/BBMB201/ Trypsin_enzymatic_activity.html. The catalytic mechanism takes place at the nanoscale and this is really a nanocatalyst. 

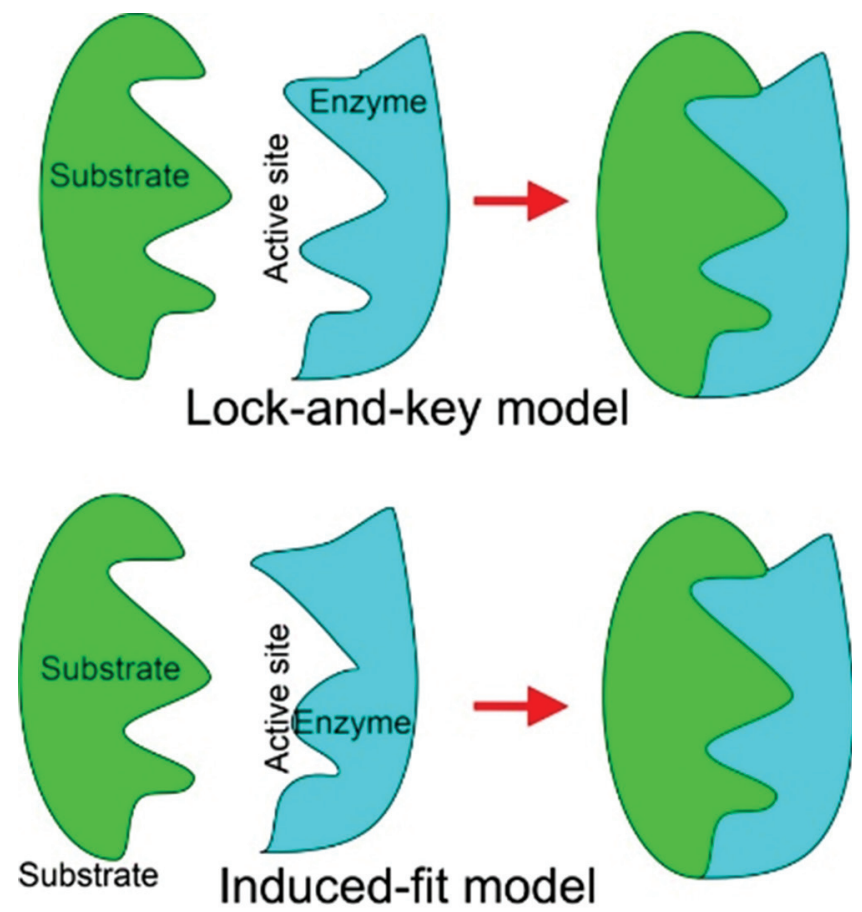

Figure 124. The principle of the lock-and-key and induced-fit models are schematically shown.

\section{Designing nanocatalysts}

Catalytic activity is based not only on chemical formula, but also on structure and the way the catalyst is used. Often, in the past, catalysts have been found experimentally by trial and error methods. Today there is a better understanding of the catalytic mechanisms. Coupling theory and experiment and taking advantage of modern computational capabilities allows today's scientists to develop catalysts faster and more efficiently.

The final goal of catalysis design is to be able to theoretically design new catalysts for chemical or biological applications with catalytic properties better than those already existing. This requires a good understanding of the basic interface mechanism between reactants and the catalyst.

For the simplest catalytic cycle needed to get a chemical or biological reaction to occur one can write:

Reactants + Catalyst $\rightarrow$ Activated Complex

Activated Complex $\rightarrow$ Products + Catalyst 


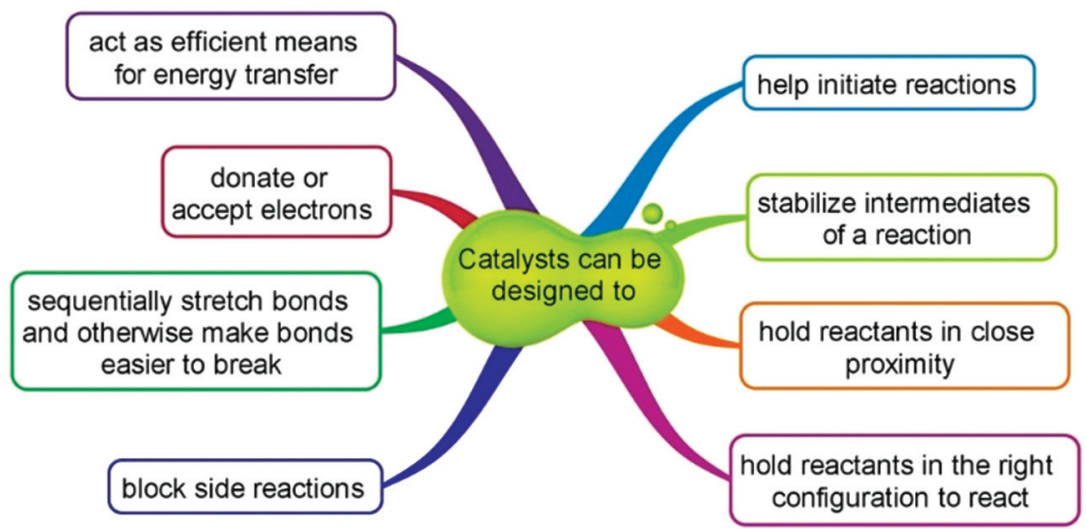

Figure 125. Characteristics of a useful catalyst. Figure based on the presentation of R.I.Mazel, Supplemental Material For Chemical Kinetics and Catalysis, ftp://ftp.wiley.com/sci_tech_med/ chemical_kinetics/.

This shows that the catalyst is not consumed. A good catalyst for a given chemical or biological reaction must have several characteristics. The most important are displayed in figure 125 .

Nanotechnology is at the heart of modern catalysis development. There are several driving forces for developing new nanocatalysts:

- To produce high-value products at low cost with inexpensive raw materials.

- To reduce, as much as possible, the energy needed to produce these products

- To develop environmentally-friendly processes

- To meet more and more stringent environmental regulations such as REACH (Registration, Evaluation, Authorization and Restriction of Chemicals) in the European Union.

- To develop low-cost catalysts replacing catalysts using precious metal

- To favor more efficiently one reaction with respect to unwanted side reactions.

- To produce valuable byproducts rather than waste.

\section{Summary}

Catalysis is an essential domain in the synthesis and transformation industry. A catalyst decreases the activation energy necessary to perform the 
reaction and increases the rate of a reaction. It provides the ability to carry out chemical and biological reactions that would be either impossible or difficult to realize in normal experimental circumstances. Biological mechanisms governing life processes are heavily based on the use of enzymes, natural catalysts. Catalytic activity depends very much on the contact surface between the catalyst and the reactants. For that reason, nanostructuration of a catalyst can be very important. The design and creation of new nanocatalysts offers revolutionary new possibilities. Not only are nanocatalysts more efficient than microcatalysts but they will also open possibilities to facilitate a much wider range of chemical and biological reactions. 